\title{
ACTIVIDAD DE Liriomyza huidobrensis DE ACUERDO A LAS DIVERSAS HORAS DEL DÍA Y TIPOS DE MUESTREO EN RELACIÓN A LA FENOLOGÍA DEL CULTIVO DE PAPA (Solanum tuberosum) ${ }^{1}$
}

\author{
Yannery Gómez ${ }^{2}$, Carlos Rodríguez ${ }^{3}$
}

\begin{abstract}
RESUMEN
Actividad de Liriomyza huidobrensis de acuerdo a las diversas horas del día y tipos de muestreo en relación a la fenología del cultivo de papa (Solanum tuberosum). Esta investigación se realizó en la zona norte de Cartago, a una altitud de 2320 m.s.n.m., de agosto a noviembre de 1990, donde se dieron los siguientes objetivos: 1. Determinar el tipo de muestreo según la edad del cultivo, que sirva para monitorear poblaciones del adulto de Liriomyza huidobrensis (Blanchard) y 2. Observar la actividad diaria de L. huidobrensis a través del día y durante un ciclo del cultivo de Solanum tuberosum. Las trampas utilizadas fueron: red entomológica a $90^{\circ}$ y $180^{\circ}$, conteo por minuto, trampa de galón plástico amarillo cubierto con grasa y tarjetas plásticas amarillas cubiertas con grasa a tres niveles de altura. Se encontró diferencia altamente significativa entre todas las trampas. Cada una, ayuda a predecir comportamientos particulares de la mosca en algún momento dado. La red $180^{\circ}$ captura moscas que se encuentran posadas y sobrevolando el cultivo. Con la red $90^{\circ}$ se capturan moscas desde la base hasta la parte superior de la planta ayudando a predecir el movimiento vertical de la mosca en la planta y con el conteo por minuto se da un estimado del número de moscas sobre las hojas. Las tarjetas amarillas ayudan a predecir la población de moscas en cada estrato (alto, medio y bajo) de la planta de papa. En el periodo de prefloración a floración se encontró la mayor población de $L$. huidobrensis con la trampa de galón y tarjetas amarillas.
\end{abstract}

\begin{abstract}
Activity of Liriomyza huidobrensis at different hours and type of sampling in relation to the phenology of the potato (Solanum tuberosum) crop. The assay was conducted on the northern zone of Cartago, Costa Rica, at an altitude of 2320 masl, from August to November of 1990. The objectives of the trial were: 1 - to determine the type of sampling, according to the crop's age, which can be used for monitoring adult populations of Liriomyza huidobrensis (Blanchard) and 2- to observe the daily activity of $L$. huidobrensis throughout the day and during one crop's cycle of Solanum tuberosum. The traps used were: entomological net at $90^{\circ}$ and $180^{\circ}$, count per minute, greased one gallon plastic jugs and yellows cards at three different heights. There were highly significant differ-ences among all the traps and, each one, helped to forecast particular behaviors of the flies at a given moment. The $180^{\circ}$ net captures flies standing on or flying over the crop. The $90^{\circ}$ net captures flies from the base to the top of the plant, helping us to forecast their vertical movement on the plant and the count per minute gives us an estimate of the number of flies on the leaves. The yellow cards help us to forecast the fly population at each stratum (high, medium and low) of the potato plant. The largest population of L. huidobrensis was found from the pre-blooming to the blooming stage with the plastic jugs and yellow cards.
\end{abstract}

\section{INTRODUCCION}

La papa es atacada por muchos insectos y en los últimos años, el "minador de las hojas" Liriomyza huidobrensis (Blanchard) se ha constituido en una plaga difícil de controlar. Este insecto también representa un gran problema en cultivos tales como, las flores de exportación principalmente crisantemos y yerberas (Price 1982), en cultivos hortícolas en los que sobresalen: apio, cebolla, vainica, lechuga, papa, remolacha y tomate (Comité técnico de Liriomyza, 1990).

Muchos investigadores han considerado que el uso de trampas es una manera aceptable e importante para el

\footnotetext{
1 Presentado en la XL Reunión Anual del PCCMCA en Costa Rica, América Central. 13 al 19 de marzo, 1994.

2 Departamento de Fitoprotección, Ministerio de Agricultura y Ganadería. San José, Costa Rica.

3 Associate Professor, Crop and Soil Sciences, Mich. State Univ., E. Lansing, MI 48824-1325.
} 
control de una plaga, por ejemplo Parrella y Jones (1984 y 1985), Robin y Mitchell (1987), Chandler (1985). Zehnder y Trumble (1985), Chapes y Raman (1987), Rodríguez et al. (1990) han utilizado las trampas de recipientes plásticos de color amarillo. Han probado ser bastante efectivas en el monitoreo de las especies de Liriomyza, en los distintos cultivos.

Por otra parte la red entomológica es otro tipo de muestreo muy usado en la captura de insectos, López (1986) la utilizó para la captura de adultos de Papión godmani (picudo del ejote). Los entomólogos han usado por mucho tiempo las redes para capturar insectos, pero su eficiencia puede variar con la especie, los diferentes hábitat y aún con los diferentes estilos de golpear con la red (Ruesink y Kogan, 1975).

Otro método de muestreo es el "levante" donde el investigador dedica tiempo en el campo para observar y cuantificar el hábitat y algunas veces se perturba el follaje (Andrews, 1986). El conteo de adulto posados sobre las hojas por simple observación o conteo visual fue probado por Schuster y Beck (1983) dado que es más fácil y se hace en menos tiempo.

Matin et al. (1984) comenta que el conteo directo es eficiente para estimar el tamaño de poblaciones como por ejemplo Diabrotica sp. en cualquier época de la edad del cultivo de maíz. Schuster y Beck (1983) trabajaron con el sistema visual con Liriomyza en tomate y concluyeron que el método es de fácil acceso y requiere de poco tiempo, porque se puede considerar este tipo de muestreo, junto con el de la trampa pegajosa, adecuado para tener idea del comportamiento del insecto en su oviposición y actividad de vuelo.

Debido al problema ocasionado por la mosca minadora y a la falta de estudios ecológicos básicos se realizaron los siguientes objetivos:

1. Determinar el tipo de muestreo según la edad del cultivo, que sirva para monitorear poblaciones del adulto de L. huidobrensis y poder así efectuar un mejor control de éste en el cultivo de papa.
2. Observar la actividad diaria de L. huidobrensis a través del día y durante un ciclo del cultivo de Solanum tuberosum.

\section{MATERIALES Y MÉTODOS}

\section{Tipos de muestreo según la fenología del cultivo de papa}

Este experimento se realizó del 24 de agosto al 28 de noviembre de 1990 en la Estación Carlos Durán del Ministerio de Agricultura y Ganadería, que pertenece al Cantón de Tierra Blanca de la provincia de Cartago, aproximadamente a 2320 m.s.n.m. Esta zona se ubica en el Macizo Irazú Turrialba y se clasifica como Bosque Tropical Premontano bajo, según Holdridge (1979).

Los tipos de muestreo se evaluaron durante la fenología del cultivo Figura 1, para ello se realizaron observaciones durante cada hora del día y durante el ciclo del cultivo con observaciones cada 15 días. Las evaluaciones se realizaron desde el momento que emergieron las primeras plantas hasta la cosecha. Para realizar este estudio se sembró semilla de papa c.v. Atzimba en una parcela de diez metros de largo por cuatro metros de ancho con cuatro surcos, y se subdividió en cuatro subparcelas con aproximadamente 50 plantas. Alrededor del campo donde se trabajó, había sembradas plantas de papa de la c.v. Atzimba y otras variedades; al oeste estaba rodeado por monte.

Para efectos de este estudio se dividió la edad del cultivo en tres partes: de siembra a prefloración (0-60 días), prefloración a floración completa (60-75 días) y de floración a cosecha (75 - 90 días).

En esta investigación se evaluaron los siguientes tipos de muestreo:

1- Se escogió un surco al azar y por simple observación se contó adultos posados en las plantas, durante un minuto, esto porque este método es fácil de utilizar y por considerar que esta mosca pasa mucho tiempo 


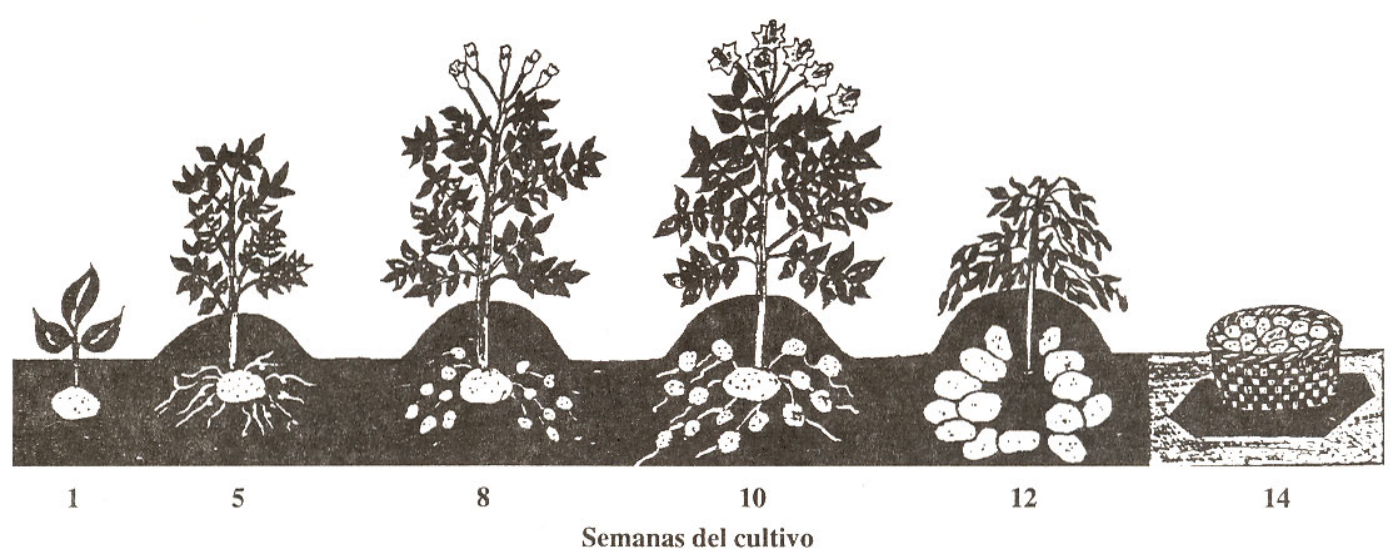

Fig. 1. Desarrollo de la planta de papa c.v. Atzimba, Llano Grande, Segura (1991).

posada sobre las hojas, ya sea para ovipositar o para alimentarse.

2- Se colocó una trampa de recipiente plástico de color amarillo con un soporte de madera de $0,11 \mathrm{~m}^{2}$ de superficie de exposición, y 3,78 litros de volumen, cubierto con grasa transparente Pennzoil 707 L. Este método de muestreo fue utilizado por Rodríguez et al (1991). La trampa se ubicó sobre la parte superior del cultivo durante una hora.

Luego se contaron los adultos L. huidobrensis adheridos en la trampa. Posteriormente se dejó la misma trampa y se hacían conteos cada ocho días. La trampa de galón la recomienda el Ministerio de Agricultura y Ganadería por lo que se utilizará para comparar con el resto de los métodos.

3- Se utilizó una red entomológica de manta con un diámetro de boca de $33 \mathrm{~cm}$. Esta se pasó a $90^{\circ}$ tres veces entre los surcos y las plantas (de la base hacia arriba).

4- Otro sistema fue la red $180^{\circ}$, pasándola tres veces encima del cultivo. Se contaron posteriormente los adultos capturados de L. huidobrensis.

5- Tarjetas amarillas, que son del mismo material que el galón, con la misma grasa ya señalada pero de un tamaño aproximado de $10 x 15 \mathrm{~cm}_{-}$. Se utilizó para monitoreo, por tener un área menor y porque se quería saber si había diferencia en la captura de la mosca en los tres estratos a saber (arriba, medio y abajo) en la planta de observación. También se comparaba capturas dentro de la planta (o sea entre las hojas) como fuera de la planta (entre surcos). Estas tarjetas fueron adheridas a un poste de madera a tres distintas alturas sobre el suelo.

\section{Actividades de Liriomyza huidobrensis (Blanchard) de acuerdo a las diversas horas del día y la fenología del cultivo de papa}

Se realizaron observaciones de la actividad de Liriomyza cada 15 días en la misma parcela mencionada en el punto 2,1 ubicada en la Estación Carlos Durán. Desde las 6 a.m hasta las 6 p.m., durante un ciclo del cultivo de la papa Solanum tuberosum. Del 13 de setiembre hasta noviembre de 1990.

La actividad de la mosca fue evaluada con todas las trampas que se tenían en estudio, y se contaron los adultos capturados de la mosca minadora con cada una de las trampas. Además se señalaron los eventos importantes en el desarrollo del cultivo de papa, que son de emergencia hasta inicio de floración (0-60 días), de prefloración hasta floración completa (60-75 días) y de floración completa a cosecha (75 - 90 días). 
Para determinar el método de muestreo más adecuado, la actividad de la mosca minadora y las horas y fechas de mayor abundancia de esta especie, se hizo un análisis de variación y la prueba de Tukey en los casos necesarios; se calculó la media, desviación estándar, coeficiente de variación (en donde se consideran: excelentes si es menor de un 5\%, muy bueno si se encuentra entre un $5 \%$ y un $10 \%$, aceptable si es menor de un $25 \%$ ) precisión y correlaciones.

Para la relación entre los distintos tipos de muestreo que se usaron, se utilizaron las correlaciones de Spearman, el análisis estadístico fue realizado con el programa MSTAT.

\section{RESULTADOS Y DISCUSIÓN}

\section{Actividad de Liriomyza huidobrensis a las diversas horas del día y tipos de muestre o en relación a la fenología del cultivo de papa}

En el Cuadro 1 se dan los resultados del coeficiente y variación y la variación relativa, donde se demuestra que hay diferencias en la captura de adultos de $L$. huidobrensis entre las trampas.

Considerando que hubo diferencias significativas en los análisis, se determinó realizar la prueba de Tukey (Cuadro 2) con el fin de saber, cual trampa tuvo la mayor captura de adultos de L. huidobrensis; a cual hora u horas se dio esa mayor captura y en cual de los períodos estudiados se da esa mayor población.

En el estudio del ciclo del cultivo de papa, se dieron tres etapas muy marcadas, se realizó la prueba del coeficiente de variación y la variación relativa, en las tres etapas por separado en cada una de las trampas.

\section{ETAPA: Emergencia a prefloración (primeros 60 días)}

En el caso de la red entomológica $180^{\circ}$ y $90^{\circ}$, el conteo por minuto y la trampa de galón, los resultados se encuentran en el (Cuadro 1), las trampas son aceptables ya que en el porcentaje de aprobación (descrito en análisis de datos) para el uso de estas trampas, se encuentran en el rango de excelente en el caso del coeficiente de variación y los valores de la variación relativa fueron muy bajos.

La red entomológica a $90^{\circ}$ fue la que obtuvo el valor más bajo, esta trampa captura los insectos que se encuentran en dos actividades distintas, como son los que se encuentran posadas en las hojas de la planta de papa y los que sobre vuelan en forma vertical a la planta; también debe notarse en el Cuadro 1 que el promedio mayor de captura de adultos, se da con esta trampa.

En segundo término se encuentra la red $180^{\circ}$, que captura aquellos insectos que sobrevuelan y se encuentran posadas sobre la parte superior de la planta y es el segundo en mayor captura promedio de adultos de la mosca minadora.

En tercera posición se encuentran el conteo por minuto, (que son aquellos insectos que se encuentran posadas sobre las hojas) y la trampa de galón (que captura a los insectos que logra atraer), ambos tienen también el mismo promedio en captura de moscas (Cuadro 1).

En el conteo por minuto L. huidobrensis (Figura 2), el insecto tiende a tener un comportamiento influido por los cambios de temperatura (Figura 3).

La actividad de la mosca con la trampa de galón amarillo disminuye cuando la temperatura es alta (más de $24^{\circ} \mathrm{C}$ ) Figura 4, en este tipo de trampa se mostró la mayor actividad de 7 a 8 de la mañana y a las 16 horas del día.

En el caso de la trampa de tarjetas amarillas afuera (Figura 5), en la parte superior de la planta, fue la más aceptable, porque se dió una gran captura de moscas. No así en la posición media y baja de afuera y la trampa de tarjetas adentro en los tres niveles (Figura 5 y 6), los resultados dieron que no son aceptables estas trampas.

En el rango de clasificación de la prueba de Tukey (Cuadro 2), se encontró que en este mismo periodo la red entomológica a $180^{\circ}$ se encuentra en el primer nivel de clasificación, es posible que esta diferencia en la 
Cuadro 1: Coeficiente de variación (C.V) y presición (V.R) de temperatura, humedad relativa y captura de L. huidobrensis con los diferentes métodos de muestreo. Estación Carlos Durán.Cartago. Agosto a noviembre de 1990.

\begin{tabular}{|c|c|c|c|}
\hline VARIABLES & PROMEMDIO & C.v. & V.R. \\
\hline \multicolumn{4}{|c|}{ EMERGENCIA A PREFLORACION ( 0-60 DIAS) N=24 } \\
\hline TEMPERATURA & 17,0 & 9,23 & 2,66 \\
\hline HUMEDAD REL. & 68,2 & 4,01 & 1,16 \\
\hline RED $180^{\circ}$ & 50,2 & 9,14 & 2,63 \\
\hline RED $90^{\circ}$ & 57,5 & 6,71 & 1,93 \\
\hline CONTEO 1 MIN. & 45,0 & 9,96 & 2,87 \\
\hline TRAMPA GALON & 45,0 & 9,96 & 2,87 \\
\hline TARJ.ADEN.ARR. & 6,7 & 22,1 & 1,30 \\
\hline TARJ.ADEN.MED. & 1,1 & 110,9 & 31,8 \\
\hline TARJ.ADEN.ABA. & 2,3 & 68,7 & 20,0 \\
\hline TARJ.AFUE.ARR. & 4,1 & 46,8 & 12,2 \\
\hline TARJ.AFUE.MED. & 2,5 & 58,0 & 16,8 \\
\hline TARJ.AFUE.ABA. & 1,3 & 93,8 & 26,9 \\
\hline \multicolumn{4}{|c|}{ PREFLORACION A FLORACION ( 60-75 DIAS) N=24 } \\
\hline TEMPERATURA & 18,9 & 11,53 & 3,33 \\
\hline HUMEDAD REL. & 71,2 & 5,14 & 1,48 \\
\hline RED $180^{\circ}$ & 24,1 & 14,6 & 4,19 \\
\hline RED $90^{\circ}$ & 16,9 & 14,0 & 4,02 \\
\hline CONTEO 1 MIN. & 18,2 & 24,0 & 6,98 \\
\hline TRAMPA GALON & 124,8 & 7,64 & 2,20 \\
\hline TARJ.ADEN.ARR. & 61,3 & 4,24 & 3,57 \\
\hline TARJ.ADEN.MED. & 18,3 & 25,0 & 7,21 \\
\hline TARJ.ADEN.ABA. & 6,0 & 42,0 & 12,0 \\
\hline TARJ.AFUE.ARR. & 57,0 & 13,1 & 3,72 \\
\hline TARJ.AFUE.MED. & 29,5 & 17,9 & 5,15 \\
\hline TARJ.AFUE.ABA. & 6,9 & 41,1 & 43,1 \\
\hline \multicolumn{4}{|c|}{ FLORACION A COSECHA ( 75-90 DIAS) $\quad \mathrm{N}=24$} \\
\hline TEMPERATURA & 18,5 & 11,9 & 3,45 \\
\hline HUMEDAD REL. & 75,0 & 5,83 & 1,68 \\
\hline RED $180^{\circ}$ & 10,0 & 25,2 & 7,27 \\
\hline RED $90^{\circ}$ & 8,2 & 27,80 & 8,03 \\
\hline CONTEO 1 MIN. & 8,1 & 34,2 & 9,87 \\
\hline TRAMPA GALON & 93,5 & 8,82 & 2,55 \\
\hline TARJ.ADEN.ARR. & 30,1 & 14,2 & 4,10 \\
\hline TARJ.ADEN.MED. & 18,2 & 21,3 & 6,14 \\
\hline TARJ.ADEN.ABA. & 9,2 & 31,2 & 9,02 \\
\hline TARJ.AFUE.ARR. & 43,8 & 12,76 & 3,82 \\
\hline TARJ.AFUE.MED. & 30,6 & 16,7 & 4,82 \\
\hline TARJ.AFUE.ABA. & 16,1 & 25,9 & 7,49 \\
\hline
\end{tabular}


Cuadro 2. Prueba de Tukey, en el ciclo del cultivo de papa con $\mathrm{P} \leq 0,05$. Ordenados en el rango de mayor a menor número de adultos capturados de moscas minadoras. Utilizando las distintas trampas, en los tres periodos estudiados, durante un día de observación. Se dan solo los ocho primeros resultados. $N=72$. Estación Carlos Durán, Cartago. 1990.

PROM. CAPTURA 2

\section{TRAMPAS VS PERIODOS}

TARJ.AMAR.AFUE

RED $180^{\circ}$

TARJ.AMAR.AFUE

TARJ.AMAR.AFUE

RED $180^{\circ}$

RED $90^{\circ}$

TRAMPA DE GALON

RED $180^{\circ}$ "

$\begin{array}{lc}\text { PREFLORACION A FLORACION } & 88 \mathrm{~A} \\ \text { EMERGENCIA A FLORACION } & 50 \mathrm{~A} \\ \text { EMERGENCIA A FLORACION } & 9 \mathrm{~A} \\ \text { FLORACION A COSECHA } & 90 \mathrm{AB} \\ \text { FLORACION A COSECHA } & 24 \mathrm{AB} \\ \text { PREFLORACON A FLORACION } & 17 \mathrm{AB} \\ \text { EMERGENCIA A PREFLORACION } & 45 \mathrm{ABC} \\ \text { PREFLORACION A FLORACION } & 24 \quad \mathrm{~L}\end{array}$

PERIODOS VS HORAS

FLORACION A COSECHA

FLORACION A COSECHA

FLORACION A COSECHA

EMERGENCIA A PREFLORACION

9 A.M

A

8 A.M

A

10 A.M

$\mathrm{AB}$

FLORACION A COSECHA

9 A.M

$\mathrm{AB}$

FLORACION A COSECHA

4 P.M

$\mathrm{AB}$

10 A.M

$\mathrm{AB}$

PREFLORACION A FLORACION

9 A.M

$\mathrm{AB}$

FLORACION A COSECHA

2 P.M

$\mathrm{AB}$

FLORACION A COSECHA "

11 A.M

$\mathrm{F}$

\section{TRAMPAS VS HORAS}

TRAMPA DE GALON AMAR.

7 A.M

$\mathrm{A}$

TRAMPA DE GALON AMAR.

8 A.M

$\mathrm{AB}$

TRAMPA DE GALON AMAR.

9 A.M

$\mathrm{AB}$

TRAMPA DE GALON AMAR.

2 P.M

$\mathrm{AB}$

TRAMPA DE GALON AMAR.

$12 \mathrm{M}$.

$A B$

TARJ. AMAR.AFUERA

7 A.M

$\mathrm{ABC}$

TARJ. AMAR.AFUERA

9 A.M

$\mathrm{ABC}$

TRAMPA DE GALON AMAR. "

1 P.M

$\mathrm{F}$

1/ Ultimo valor en la lista de rangos de la prueba de Tukey

2/ Letras de significancia 


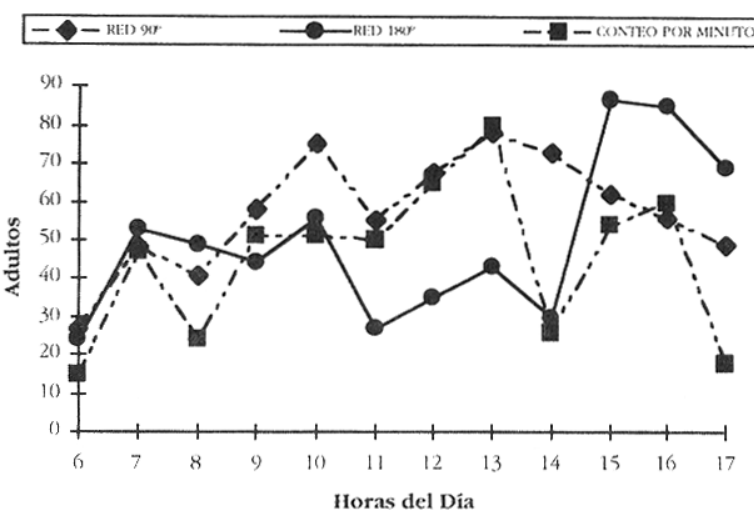

Fig. 2. Métodos de muestreo red $90^{\circ}$, red $180^{\circ}$, conteo por minuto. Estación Carlos Durán. Cartago. 1990.

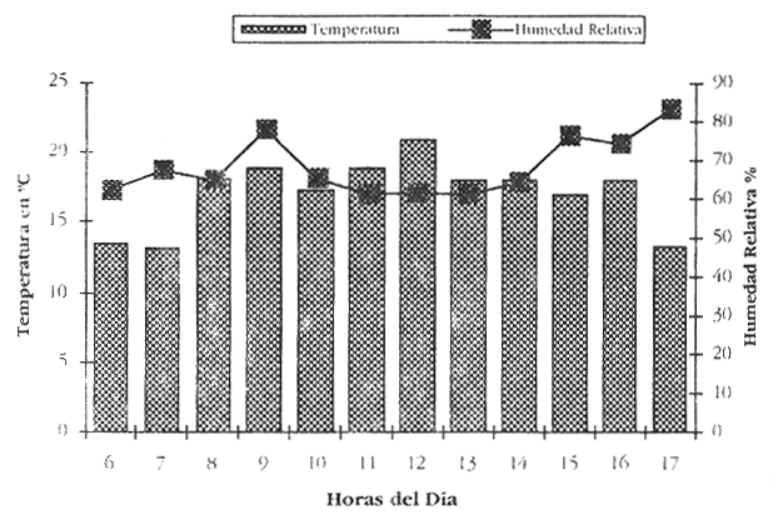

Fig. 3. Temperatura y humedad relativa. Estación Carlos Durán. Cartago. 1990.

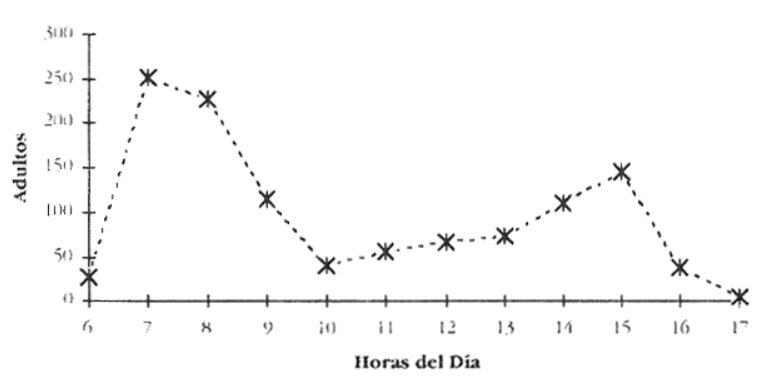

Fig. 4. Captura de L. huidobrensis con trampa galón amarillo. Estación Carlos Durán. Cartago. 1990.

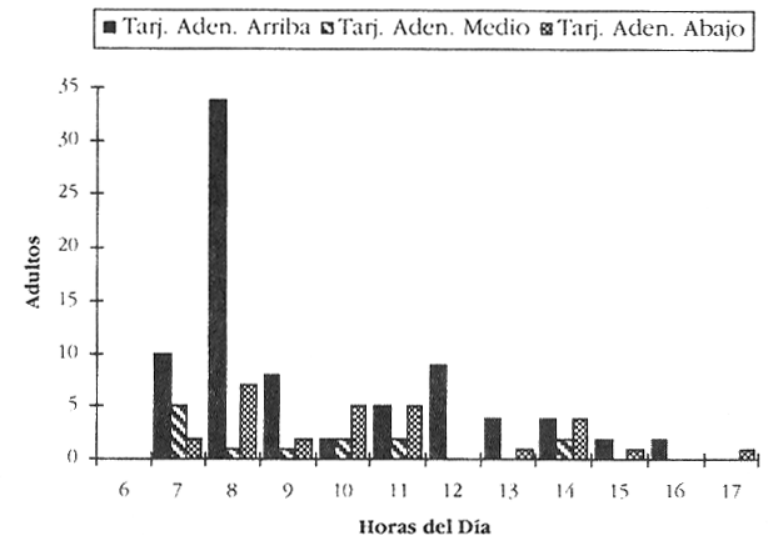

Fig. 5. Capturade L. huidobrensis en trampaubicada a tres niveles arriba, medio y abajo, en la planta de la papa. Adentro. Estación Carlos Durán, Cartago. 1990.

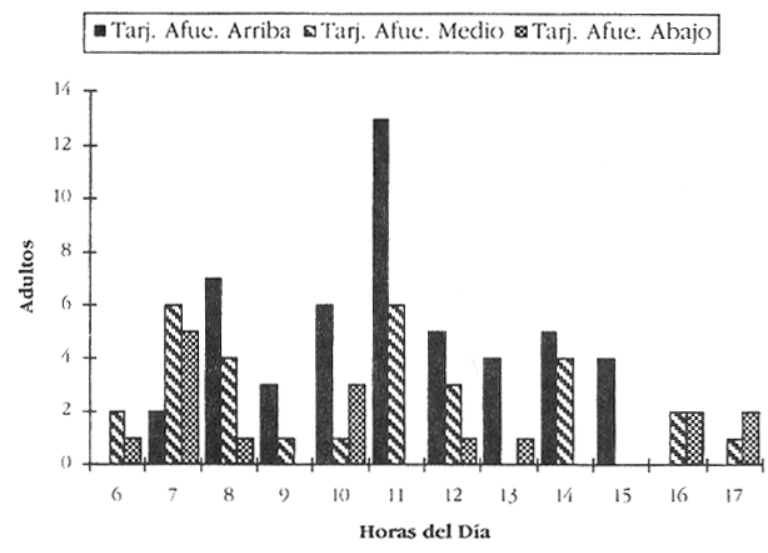

Fig. 6. Captura de L. huidobrensis en trampa ubicada a tres niveles arriba, medio y abajo, en la planta de la papa. Afuera. Estación Carlos Durán, Cartago. 1990.

clasificación de trampas con respecto al coeficiente de variación, donde la red a $90^{\circ}$ está en primer término, se deba a que en esta parte del periodo las plantas están en continuo crecimiento. La red a $90^{\circ}$ en los distintos días de evaluación, recorría alturas diferentes, conforme fuera creciendo la planta.

La tarjeta amarilla en la parte superior de afuera se encuentra en el segundo nivel de clasificación según Tukey (Cuadro 2) y la trampa de galón amarillo en el tercer nivel. 
Con la trampa de tarjetas amarillas se evaluaron tres estratos, se realizó el análisis de correlación de Sperman (Cuadro 3), tanto las tarjetas dentro y fuera de la plantación y entre cada uno de los estratos, los resultados demostraron, que si hay una gran asociación entre colocar la trampa dentro de la planta o fuera de ella, y que conforme más abajo en el estrato esta correlación presenta un valor menor.

Cuadro 3. Correlación de Spearman para determinar la correlación entre colocar la tarjeta amarilla fuera de la planta o dentro de ella y entre los tres estratos (alto, medio y bajo de la planta). $\mathrm{P}=0.000$ y $\mathrm{N}=72$. Estación Carlos Durán, Cartago. De agosto a noviembre 1990.

\begin{tabular}{lrrr}
\hline Localización & \multicolumn{2}{c}{ Trampa localizada } \\
\cline { 2 - 3 } & $\begin{array}{c}\text { Dentro de la } \\
\text { planta de papa }\end{array}$ & $\begin{array}{r}\text { Afuera de la } \\
\text { planta de papa }\end{array}$ \\
\hline & Arriba & Medio & Abajo \\
Arriba & 0,94 & & \\
$\begin{array}{l}\text { Medio } \\
\text { Abajo }\end{array}$ & & 0,89 & \\
\hline
\end{tabular}

Los resultados del Cuadro 1 demuestran, que el número promedio en captura de adultos de la mosca minadora, considerando solo las trampas de tarjetas amarillas, se da en la parte de arriba.

Es posible que esto se deba a que la intensidad de luz varía en forma vertical en la planta de papa y que este factor haga que el adulto de L. huidobrensis busque el estrato superior. Además es en la parte superior de la planta, donde se tiene el tejido más nuevo que resulta más atractivo para el adulto de L. huidobrensis. Gunter (1988) indica que para L. trifolii las hojas jóvenes son preferidas para alimentación y hojas mayores para oviposición.

La temperatura es otro factor importante en la actividad de los adultos de la mosca minadora. Para determinar si la temperatura tenía alguna influencia en la captura de moscas con las distintas trampas, se realizó un análisis de variación (Cuadro 4) donde se encontró que las trampas tuvieron resultados altamente significativos.
La Figura 3 muestra que al ir aumentando la temperatura, la captura en las trampas aumentan también, hasta las 8 a.m.; de aquí en adelante hay cambios de temperatura, situación que se da también con la red $180^{\circ}$, $90^{\circ}$ y conteo por minuto.

Con la trampa amarilla tanto tarjetas como galón, se observan cambios bruscos en la captura de adultos de Liriomyza.

Se mostró en el (Cuadro 4) que la temperatura tiene influencia con las trampas, y en la Figura 2 se muestra como se da esta influencia con la trampa de la red entomológica a $90^{\circ}$ y $180^{\circ}$ donde se observa una variación parecida con la captura de las moscas y los cambios de temperatura, en el Cuadro 1 el coeficiente de variación de estos dos métodos son los más bajos. En el Cuadro 2 se da en el rango de mayor a menor captura y la red $180^{\circ}$ en este periodo, está en segunda posición.

Se observó la mayor captura de las moscas, en las tarjetas amarillas ubicadas en la parte superior (Figura 5 y 6), principalmente con la tarjeta adentro donde se da un pico de máxima actividad a las 8 a.m. y en los demás estratos las oscilaciones de captura a través del día son muy parecidos.

En este periodo las capturas promedios de todas las trampas son muy parecidas (Cuadro 1).

Con la humedad relativa (Figura 3), la correlación con la temperatura es alta pero negativa sucediendo que cuando la temperatura sube, la humedad es baja.

\section{ETAPA: Prefloración a floración (60-75 días)}

En este periodo se da la mayor captura de adultos de Liriomyza (Cuadro 1) con todas las trampas.

La plantación en este período mostró un gran deterioro por el ataque no sólo de L. huidobrensis, sino también por la infección del hongo Phytophthora infestans. 
Cuadro 4. Resultados del análisis de variación, entre la temperatura y las trampas en cada uno de los períodos estudiados. Estac. Carlos Durán, Cartago. 1990.

\begin{tabular}{|c|c|c|c|}
\hline VARIABLES & C.M & VALORES F & PROBABILIDAD \\
\hline \multicolumn{4}{|c|}{ EMERGENCIA A PREFLORACION N=24 G.L. $=1$} \\
\hline $\operatorname{Red} 180^{\circ}$ & 6,570 & 29,01 & 0,0000 \\
\hline $\operatorname{Red} 90^{\circ}$ & $9,805 \mathrm{E}+03$ & 84,97 & 0,0000 \\
\hline Conteo minuto & $4,707 \mathrm{E}+03$ & 22,86 & 0,0001 \\
\hline Trampa galón & $3,785 E+04$ & 12,43 & 0,0019 \\
\hline Tarj.aden arr. & 650,0 & 14,24 & 0,0010 \\
\hline Tarj.aden med. & $1,534 \mathrm{E}+03$ & 364,78 & 0,0000 \\
\hline Tarj.aden aba. & $1,304 \mathrm{E}+03$ & 223,60 & 0,0000 \\
\hline Tarj.afue arr. & $1,013 \mathrm{E}+03$ & 102,89 & 0,0000 \\
\hline Tarj.afue med. & $1,275 \mathrm{E}+03$ & 240,44 & 0,0000 \\
\hline Tarj.afue aba. & $1,487 \mathrm{E}+03$ & 354,43 & 0,0000 \\
\hline \multicolumn{4}{|c|}{ REFLORACION A FLORACION N=24 G.L. $=1$} \\
\hline $\operatorname{Red} 180^{\circ}$ & 167,5 & 1,91 & 0,1805 \\
\hline Red $90^{\circ}$ & 23,21 & 0,85 & 0,3670 \\
\hline Conteo minuto & 2,407 & 0,01 & 0,9138 \\
\hline Trampa galón & $8,125 \mathrm{E}+04$ & 21,56 & 0,0001 \\
\hline Tarj.aden arr. & $1,081 \mathrm{E}+04$ & 6,46 & 0,0186 \\
\hline Tarj.aden med. & 1,815 & 0,01 & 0,9305 \\
\hline Tarj.aden aba. & 983,0 & 31,18 & 0,0000 \\
\hline Tarj.afue arr. & $8,755 E+03$ & 5,69 & 0,0261 \\
\hline Tarj.afue med. & 665,7 & 1,66 & 0,2114 \\
\hline Tarj.afue aba. & 859,2 & 19,45 & 0,0002 \\
\hline FLORACION A COSECHA & $\mathrm{N}=24 \quad$ G.L $=1$ & & \\
\hline $\operatorname{Red} 180^{\circ}$ & 429,3 & 13,23 & 0,0015 \\
\hline $\operatorname{Red} 90^{\circ}$ & 625,3 & 24,31 & 0,0001 \\
\hline Conteo minuto & 645,8 & 15,50 & 0,0007 \\
\hline Trampa galón & $3,379 \mathrm{E}+04$ & 14,48 & 0,0010 \\
\hline Tarj.aden arr. & 810,8 & 4,50 & 0,0454 \\
\hline Tarj.aden med. & $3,604 \mathrm{E}+01$ & 0,00 & 0,9640 \\
\hline Tarj.aden aba. & 564,3 & 12,26 & 0,0020 \\
\hline Tarj.afue arr. & $3,863 \mathrm{E}+03$ & 6,73 & 0,0165 \\
\hline Tarj.afue med. & 882,1 & 2,50 & 0,1279 \\
\hline Tarj.afue aba. & 41,34 & 0,25 & 0,6252 \\
\hline
\end{tabular}


En esta parte los resultados del coeficiente de variación mostrados en el Cuadro 1, las trampas de más aceptación en primer término es la tarjeta afuera arriba, la cual en comparación con las otras tarjetas es la que tiene el promedio mayor de captura.

Con la prueba de Tukey (Cuadro 2) se obtuvo que este periodo con las tarjetas a tres niveles, principalmente en la parte superior fue donde se dio la mayor captura, y que con las trampas amarillas en horas de la mañana ( 7,8 y 9) se dieron capturas altas, además con estas dos trampas se obtuvo el coeficiente de variación menor (Cuadro 1).

En la prueba de Tukey (Cuadro 2) se encuentra la trampa de galón amarillo que viene a dar un resultado parecido a la tarjeta amarilla en la parte superior y es la que mayor captura promedio tiene en esta etapa.

La captura en las trampas de las tarjetas amarillas en la parte de arriba, tanto adentro como afuera es más alta (Figura 7 y 8), disminuyó gradualmente hasta ser más baja en la parte inferior. Esto se debe a que las hojas en el nivel inferior, están sumamente atacadas por el hongo y las hojas superiores son más nuevas y sanas, además se da mayor luminosidad.

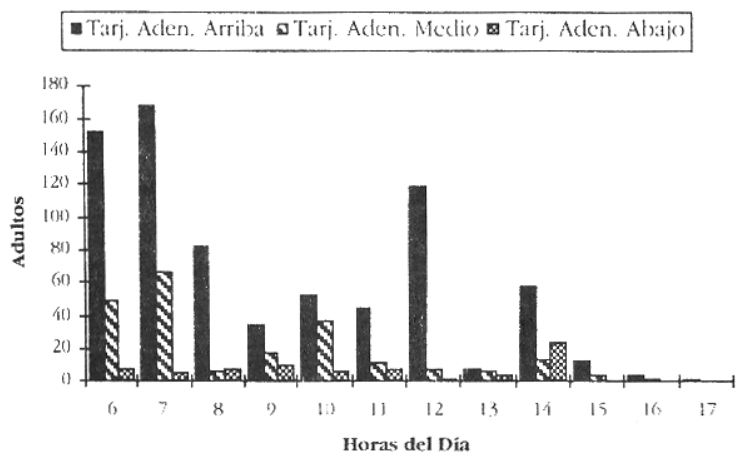

Fig. 7. Captura de L. huidobrensis con trampas ubicadas a tres niveles arriba, medio, abajo en planta de papa. Adentro. Estación Carlos Durán. Cartago. 1990.

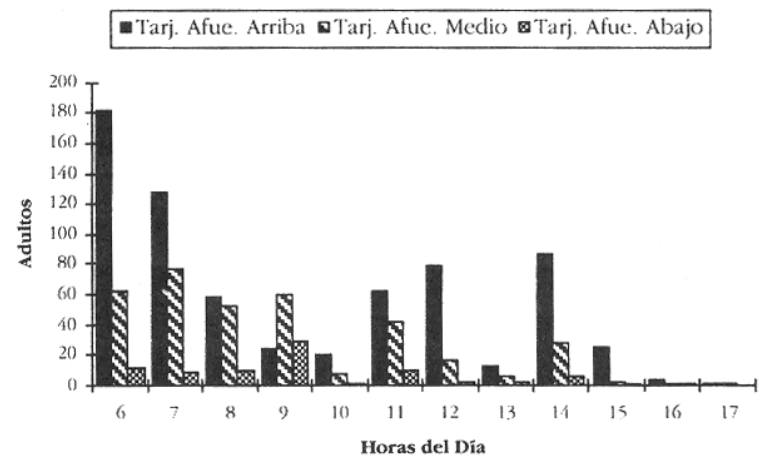

Fig. 8. Captura de L. huidobrensis con trampas ubicadas a tres niveles arriba, medio, abajo en planta de para. Afuera. Estación Carlos Durán. Cartago. 1990.

Siguiendo de mayor a menor captura de las trampas se encuentran tarjeta afuera arriba, red $90^{\circ}, 180^{\circ}$, conteo por minuto y tarjeta adentro medio y afuera medio.

La red $180^{\circ}$ (Figura 9) la trayectoria de la curva a través del día es muy parecida con la curta de la temperatura (Figura 10). En el caso de la red $90^{\circ}$ la curva lleva variaciones cortas a través de todo el día (Figura 9).

En el conteo por minuto (Figura 9) se da una captura máxima a las 7 de la mañana y baja gradualmente para mantener más o menos constante el resto del día.

En esta etapa las trampas de menor captura son la de tarjeta abajo tanto adentro como afuera (Cuadro 1).

Con la prueba de Tukey Cuadro 2, el resultado muestra que en esta parte del cultivo la trampa de tarjetas amarillas afuera se encuentra en el primer nivel y la red $90^{\circ}$ y $180^{\circ}$ en el segundo y tercer nivel respectivamente.

Con respecto a los cambios en la temperatura se encontraron diferencias de la temperatura mínima a la máxima de $14^{\circ} \mathrm{C}$. Al ser comparadas la curva de la temperatura (Figura 10) con las trampas se observa que lleva cierta semejanza con la red $180^{\circ}$ (Figura 9), no así con el resto de las trampas. 


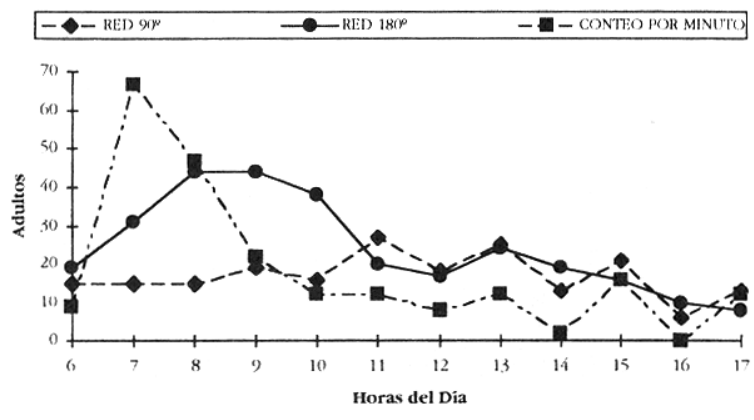

Fig. 9. Métodos de muestreo red $90^{\circ}$, red $180^{\circ}$, conteo por minuto. Estación Carlos Durán. Cartago. 1990.

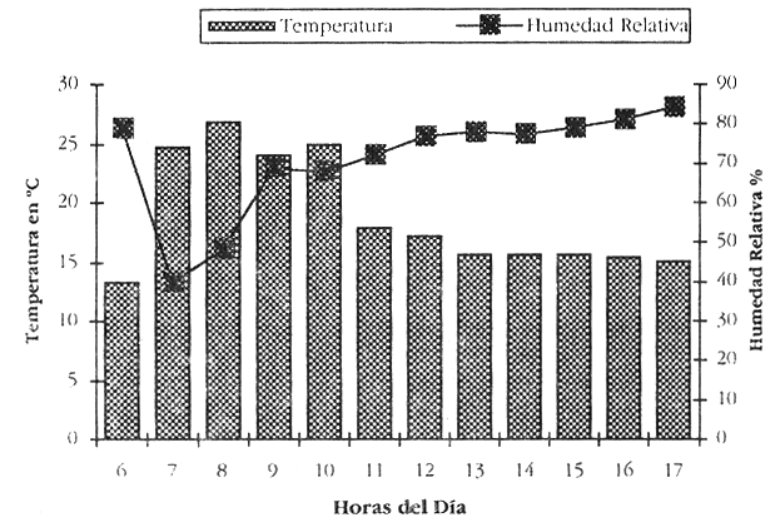

Fig. 10. Temperatura y humedad relativa. Estación Carlos Durán. Cartago. 1990.

En este periodo se encontraron semejanzas con el anterior, el comportamiento está condicionado con la temperatura y la luminosidad, a mayor temperatura (cerca de $25^{\circ} \mathrm{C}$ ) Figura 10, mayor cantidad de adultos posados en la planta (Figura 9) en proporción con la captura de la trampa de galón (Figura 11).

\section{ETAPA: Floración a cosecha (75-90 días)}

Este y el periodo anterior, son los que mostraron mayor captura de adultos de Liriomyza (Cuadro 1), muestra que los periodos son significativamente diferentes, para la captura de adultos de la mosca minadora con cada una de las trampas.

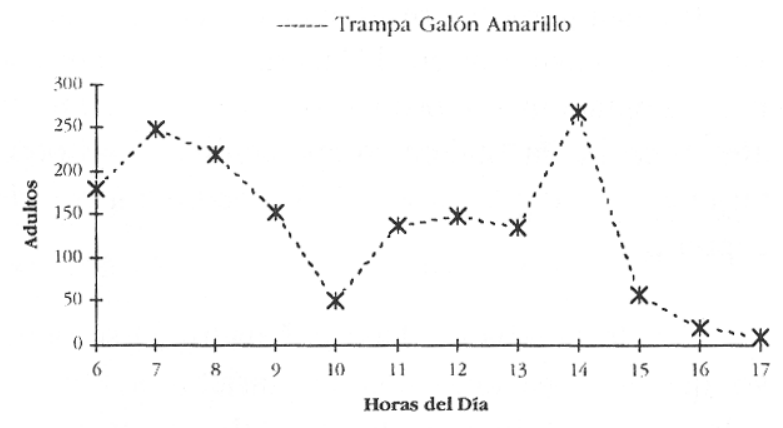

Fig. 11. Captura de L. huidobrensis con trampa galón amarillo. Periodo de prefloración y floración (60-75 días). Estación Carlos Durán. Cartago. 1990.

En este periodo el cultivo estaba gravemente afectado por el hóngo $P$. infestans además de senescente. Gran cantidad de plantas estaban caídas, ya la captura con red a $180^{\circ}, 90^{\circ}$ y conteo pcu minuto se disminuyó (Figura 12), porque existía muy poco tejido vivo, el cultivo tenía dos meses y medio.

En esta etapa se dan valores del coeficiente de variación bajos y por lo tanto de aceptación excelente, para la trampa de galón, las tarjetas afuera y adentro en la parte de arriba.

El resto de las trampas no caen en el rango de aceptación confiable, esto prodría deberse a que en este periodo, la mayoría de las plantas estan atacadas por hongos y/o senescentes, no existe substrato donde la mosca pueda posarse, por lo tanto hay mayor exposición de la trampa de galón amarilla que es de por sí atrayente dándole ventaja con respecto a las demás trampas, por lo que su captura promedio es mayor.

$\mathrm{Al}$ darse cambios bruscos de la temperatura (Figura 13), no se observa que la curva tenga alguna relación con las trampas en general, aunque en el análisis de variación dio significativo.

A pesar de que las temperaturas fueron altas Figura 13, siempre hubo población en el lugar puesto que siempre encontrará la mosca un nicho donde ocultarse y 


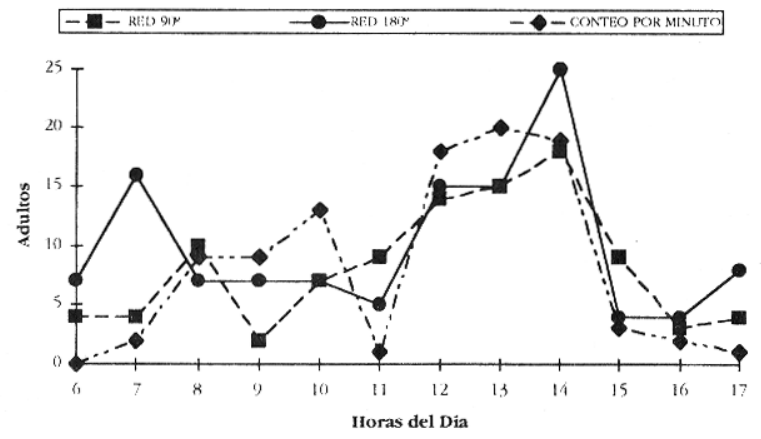

Fig. 12. Métodos de muestreo Red $90^{\circ}, 180^{\circ}$ y conteo por número. Estación Carlos Durán. Cartago. 1990.

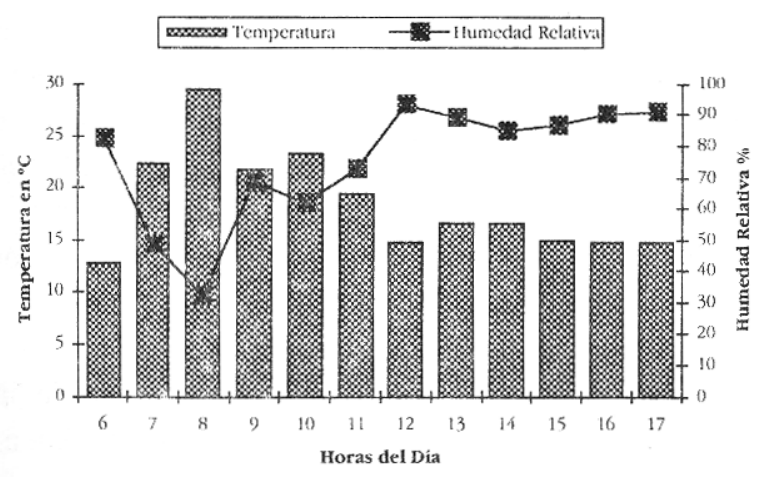

Fig. 13. Temperatura en ${ }^{\circ} \mathrm{C}$ y humedad relativa $\%$. Estación Carlos Durán. Cartago. 1990.

posarse, además encontrar parte de su alimentación. La captura en la trampa es alta, o sea hay todavía mucha actividad principalmente por el hecho de que no existe substrato donde posarse Figura 14.

Lo mismo sucedió con las tarjetas, la captura es principalmente alta arriba, Figuras 15 y 16. Este aspecto es importante ya que las trampas sirven de apoyo para tomar decisiones, pero se debe conocer la edad del cultivo y saber la importancia del follaje en ese período y no orientarse sólo por las lecturas hechas en las trampas, para que en éstas se pueden obtener capturas sin ser en ese momento la mosca minadora tan importante para el cultivo.

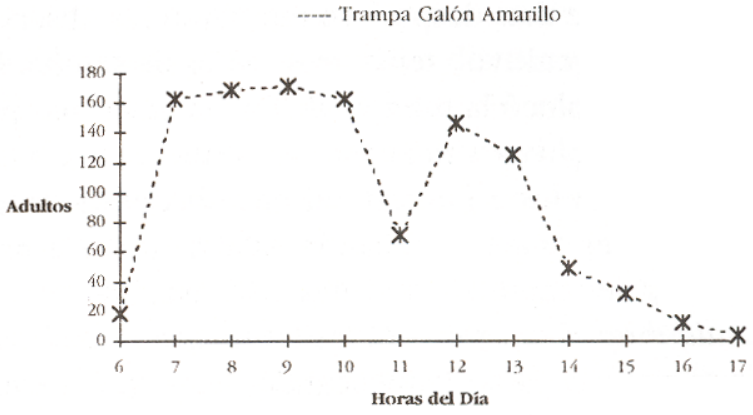

Fig. 14. Captura de L. huidobrensis en trampa de galón amarillo. Estación Carlos Durán. Cartago. 1990.

- Tarj. Aden. Arriba w'Tari. Aden. Medio a'Tarj. Aden. Abajo

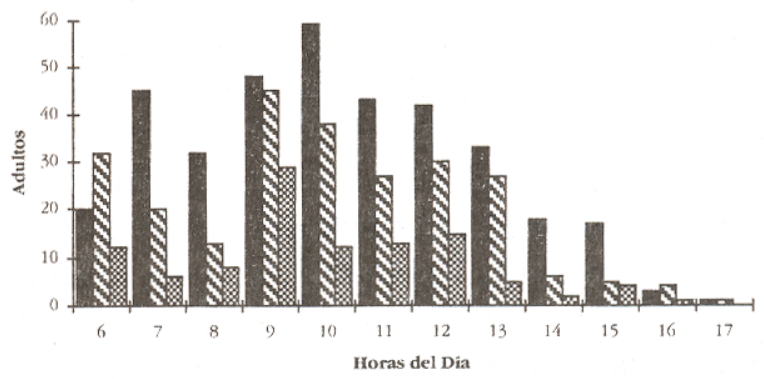

Fig. 15. Captura de Liriomyza con trampa ubicada a tres niveles arriba, medio, abajo en la planta de papa. Adentro. Estación Carlos Durán. Cartago. 1990.

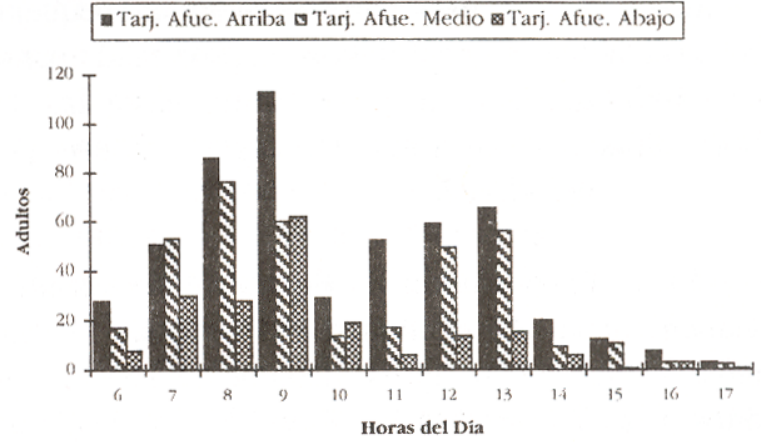

Fig. 16. Captura de Liriomyza con trampa ubicada a tres niveles arriba, medio, abajo en la planta de papa. Afuera. Estación Carlos Durán. Cartago. 1990. 
Quince días después se repitieron las observaciones, el cultivo tenía tres meses de sembrado, sólo se colocó la trampa de galón amarillo, por estar el cultivo sumamente destruido, desde las 6 a.m. hasta las 2 p.m., esta última observación, fue para saber cuánta población estaba todavía presente, se capturó en total 365 adultos de $L$. huidobrensis, el resto de las trampas no se utilizaron, porque ya no tenía sentido, hubo que aplicar herbicida para quemar el cultivo y evitar que el hongo avanzara más y atacara el tubérculo.

En este periodo resulta adecuado utilizar una gran cantidad de trampas de galón de color amarillo, para realizar una captura masiva y disminuir la población relativa presente para evitar un foco de infección no sólo para el mismo agricultor, ya que si decide sembrar nuevamente papa en un lapso corto se perjudicaría y para evitar migraciones de la mosca minadora a los campos vecinos.

Con la trampa de galón amarillo, la curva a través del día en los tres periodos es muy diferente, por ejemplo en la Figura 4 a las 7 a.m. se da una captura muy alta de casi 250 individuos y otro pico importante es a las 15 horas con una captura de 150 individuos.

En el periodo de prefloración a floración la captura es mayor (Figura 11), la curva en la mañana es muy parecida al periodo anterior obteniéndose una captura máxima a las 7 a.m. de 250 individuos, inclusive a las 10 a.m. en ambos periodos se muestra la menor captura, pero hacia el medio día en esta parte se nota un pequeño pico de actividad capturándose aproximadamente 150 individuos, sigue en aumento hasta las 14 horas donde se da la máxima captura de este periodo de 269 individuos.

En el último periodo (Figura 14) se da nuevamente una alta actividad en horas de la mañana, capturándose aproximadamente 165 individuos a las 7 a las 8 a las $9 y$ 10; y un segundo pico a las 12 de 146 individuos y luego disminuye poco a poco en el transcurso de la tarde.

Las tarjetas amarillas adentro arriba (Figuras 5, 6, 7 y 8 ), en el primer y segundo periodo tienen un comportamiento parecido a la trampa de galón, los otros dos estratos no muestran grandes diferencias. En el último periodo es en tarjetas afuera arriba la que toma las características de la curva a la trampa galón, las tarjetas adentro tiene otras oscilaciones.

Con las trampas de la red $180^{\circ}$ y $90^{\circ}$ no muestra en todo el ciclo picos importantes en el transcurso del día, se dan pequeñas fluctuaciones a través de todo el día.

Lo mismo sucede con el conteo por minuto con la diferencia que en el periodo de prefloración a floración, muestra un pico muy pronunciado a la 7 a.m. para luego seguir con pequeñas variaciones.

Al capturar moscas minadoras con estas trampas, cada una ayuda a predecir comportamientos particulares del insecto, en algún momento dado. Por ejemplo con la red $180^{\circ}$, se capturan moscas que se encuentran posadas y sobrevolando el cultivo ya que la red se desplaza por encima de la planta.

Con la red $90^{\circ}$ se capturan moscas desde la base del tallo hasta la parte superior de la planta, puede ayudar a predecir el comportamiento vertical que tiene la mosca.

Con las tarjetas amarillas a tres niveles, tanto la que se encuentra adentro (a la par de la planta), como la que se encuentra afuera (en el surco), al ser atrayente, ayudará a predecir también cual es el comportamiento de la mosca en forma vertical y determinar el estrato donde ella permanece más tiempo.

Por último con la trampa de galón plástico amarillo, al ser el amarillo atrayente para la mosca minadora, como ha sido probado por otros investigadores, como Parrella y Jones (1985) y Chandler (1985), puede servir como un método cultural de captura y da un indicio para determinar si hay moscas en la plantación y en que proporción.

Por lo tanto se consideran que todas las trampas aquí estudiadas pueden ser usadas para el monitoreo de la mosca minadora y que cada una de estas trampas según 
sea el estado vegetativo del cultivo, pueden capturar en mayor o menor medida este insecto y pueden ayudar a predecir la actividad del insecto durante el ciclo del cultivo.

Chandler (1985) y Rodríguez, et al. (1990) trabajaron con la trampa pegajosa de galón amarillo, confirman que la mosca minadora parece tener dos momentos importantes de actividad, en la mañana de 6 am hasta las 9.30 am y en la tarde de 2 pm hasta aproximadamente las 5 p.m., esta información resulta de suma importancia, porque permite programar la hora para la aplicación de los agroquímicos cuando sea necesario e informar al agricultor de estos momentos de máxima actividad de mortalidad de adultos de la mosca.

Chandler (1985) trabajó en el cultivo de pimienta midiendo la actividad de vuelo de L. trifolii con la trampa amarilla a varios niveles del suelo, midió la intensidad solar y concluyó que un rango de 1,0-90,0 mW/ $\mathrm{cm}^{2}$, que corresponde al período de horas que señalan la actividad de la mosca minadora, en este rango de luminosidad y temperaturas de $20^{\circ} \mathrm{C}$ a $25^{\circ} \mathrm{C}$, este insecto tiende a sobrevolar por el cultivo, y a temperaturas más altas, pareciera mantenerse más tiempo posada en la hoja tanto en la parte del haz como en el envés.

Vélez et al. (1980) manifiestan que a temperaturas de $26,7^{\circ} \mathrm{C}$ se da al máximo la oviposición y se da la mayor emergencia de adultos.

Se realizó la prueba de la correlación de Spearman (Cuadro 5), y se comprobó que sí había relación entre los distintos tipos de muestreo probados en esta investigación y la Humedad Relativa, contra la trampa de galón plástico amarillo.

Los resultados (Cuadro 5) muestran en cada una de las etapas estudiadas en esta investigación, que en la primera parte desde la siembra hasta la floración, existe correlación de la trampa de galón con la red $180^{\circ}$, red $90^{\circ}$, conteo por minuto, tarjetas adentro (arriba y abajo), tarjetas afuera arriba, es $>0,8$ altamente significativo, lo mismo que con la humedad relativa. Pero la asociación de la trampa con tarjetas adentro medio y tarjetas afuera medio y abajo va siendo menor comparado con las anteriores.
Por el contrario en las dos etapas siguientes prefloración a cosecha, las $\mathrm{r}$ disminuyen (Cuadro 5), con la red entomológica y el conteo por minuto, es posible que esto se deba a que en estos periódos las plantas se van poniendo senecentes sobre todo en el último periodo, estan sumamente atacadas por hongos y bacterias, esto hace que con la red entomológica la captura se más difícil, y con el conteo por minuto donde se cuentan adultos posados sobre las plantas, el conteo también se dificulte, ya que las moscas están más expuestas, la probabilidad en este caso no es significativa.

La correlación con la humedad relativa es negativa, $\mathrm{r}=-0,55$ y $-0,67$ para los periodos de pref]oración a cosecha, a mayor humedad relativa, menor captura en la trampa de galón.

La trampa pegajosa del galón amarillo tiene ciertos inconvenientes (los cuales se enumeran más adelante) que podrían disminuir la eficiencia de la trampa; si se expusiera por más de ocho días.

Estudio realizado por (Gómez, Y., et al. 1992) se evaluó la trampa de galón amarillo cada 8 días. Se concluyó que tres días de exposición de la trampa, podría ser el límite máximo, por las siguientes razones: las lluvias durante el tiempo que la trampa estuvo expuesta le van quitando efectividad a la grasa. El viento acarrea mucho polvo, ensucia la trampa que afecta también a la grasa y con ello se disminuye la captura.

Si en el cultivo de papa las poblaciones de $L$. huidobrensis son muy altas, en pocos días el galón se satura de moscas capturadas, esta excesiva población hace el con tea sumamente difícil, por lo que es necesario hacer estimados de los adultos capturados y se dejan de capturar muchas moscas. Además semejante a la red entomológica, los cambios en la distribución vertical no son cuantificados.

Pero no obstante estas desventajas, y aunque la diferencia entre las trampas es altamente significativa, se considera que la trampa de galón amarillo es más práctica en su uso por las siguientes razones: 
Cuadro 5. Correlaciones de Spearman para comparar la relación de los distintos tipos de muestreo, con respecto a la trampa de galón amarillo. En un ciclo del cultivo de papa. Estación Carlos Durán, Cartago, agosto a noviembre de 1990 .

\begin{tabular}{|c|c|c|}
\hline Tipos de muestreo & Resultados de $\mathbf{r}$ & Probabilidad \\
\hline \multicolumn{3}{|c|}{ SIEMBRA-PREFLORACION (PRIMEROS 60 DIAS) N=24 } \\
\hline Red 180 & 0,84 & $\mathrm{P}>0,01$ \\
\hline Red 90 & 0,82 & $\mathrm{P}>0,01$ \\
\hline Conteo en 1 minuto & 0,85 & $\mathrm{P}>0,01$ \\
\hline Tarjeta Adentro Arriba & 0,92 & $\mathrm{P}>0,01$ \\
\hline Tarjeta Adentro Medio & 0,66 & $\mathrm{P}>0,01$ \\
\hline Tarjeta Adentro Abajo & 0,80 & $P>0,01$ \\
\hline Tarjeta Afuera Arriba & 0,81 & $P>0,01$ \\
\hline Tarjeta Afuera Medio & 0,73 & $\mathrm{P}>0,01$ \\
\hline Tarjeta Afuera Abajo & 0,52 & $\mathrm{P}>0,01$ \\
\hline Humedad relativa & 0,75 & $P>0,01$ \\
\hline \multicolumn{3}{|c|}{ PREFLORACION - FLORACION ( 60-75 DIAS) N=24 } \\
\hline Red 180 & 0,50 & $\mathrm{P}>0,01$ \\
\hline Red 90 & $-0,05$ & $\mathrm{P}<0,05$ \\
\hline Conteo en 1 minuto & 0,22 & $\mathrm{P}<0,05$ \\
\hline Tarjeta Adentro Arrriba & 0,79 & $\mathrm{P}>0,05$ \\
\hline Tarjeta Adentro Medio & 0,63 & $\mathrm{P}<0,05$ \\
\hline Tarjeta Adentro Abajo & 0,73 & $\mathrm{P}>0,05$ \\
\hline Tarjeta Afuera Arriba & 0,83 & $\mathrm{P}<0,05$ \\
\hline Tarjeta Afuera Medio & 0,83 & $\mathrm{P}>0,05$ \\
\hline Tarjeta Afuera Abajo & 0,75 & $\mathrm{P}>0,05$ \\
\hline Humedad Relativa & $-0,55$ & $\mathrm{P}<0,05$ \\
\hline FLORACION - COSECHA ( 75 DIAS) & $\mathrm{N}=24$ & \\
\hline Red 180 & 0,22 & $\mathrm{P}<0,05$ \\
\hline Red 90 & 0,08 & $\mathrm{P}<0,05$ \\
\hline Conteo en 1 minuto & 0,47 & $\mathrm{P}>0,01$ \\
\hline Tarjeta Adentro Arriba & 0,84 & $\mathrm{P}>0,01$ \\
\hline Tarjeta Adentro Medio & 0,83 & $P>0,01$ \\
\hline Tarjeta Adentro Abajo & 0,68 & $\mathrm{P}>0,01$ \\
\hline Tarjeta Afuera Arriba & 0,86 & $\mathrm{P}>0,01$ \\
\hline Tarjeta Afuera Medio & 0,83 & $\mathrm{P}>0,01$ \\
\hline Tarjeta Afuera Abajo & 0,92 & $\mathrm{P}>0,01$ \\
\hline Humedad Relativa & $-0,67$ & $\mathrm{P}<0,05$ \\
\hline
\end{tabular}

* Parte de la tesis de grado del primer autor. Biología, UCR.

" Depto. de Fitoprotección - M.A.G. , Costa Rica.

2) Del Monte Specialty, Costa Rica. 
1- No es costosa.

2- Requiere de poco tiempo para colocarla.

3- La captura de moscas minadoras es bastante alta.

4- Se puede mantener en el lugar por varios días (con revisiones por lo menos cada cuatro días), además comparado con el sistema visual, cuantifica los insectos presentes cuando el observador está ausente, se pueden colocar por todo el terreno.

5- Esta trampa ya ha sido estudiada y utilizada por una gran cantidad de investigadores.

Por otra parte Zechnder y Trumble (1985), Chaves y Raman (1987), consideran que la trampa puede ser usada perfectamente para monitoreo de L. huidobrensis.

Parrella (1984) indica que esta técnica es muy eficiente y puede predecir un problema potencial en el cultivo.

Con la red entomológica utilizada a través de todo el ciclo del cultivo de papa, pareciera reflejar como aumenta y disminuye la población de la mosca minadora y como su comportamiento en todo el día en las tres etapas es muy diferente, por ejemplo en la Figura 2, se muestra que el comportamiento del insecto a través del día tuvo altibajos, con capturas que fueron desde 18 hasta 75 adultos de la mosca.

En la Figura 9 con la red 1800 se da una gran actividad en la mañana hasta las 10 am y luego baja, con la red 900 la captura varió desde 0 hasta 40 .

Por último en la Figura 12 se muestran cambios de captura a través de todo el día y la captura en esta etapa fue muy baja, va desde 0 hasta 18 adultos de la mosca.

Por lo tanto se recomienda usarla para efectos de monitorio, ya que puede suministrar información valiosa sobre la actividad de la mosca minadora. Por razones prácticas, ésta trampa tiene ciertas desventajas con respecto a la trampa de galón amarillo, como son: es poco práctica, en el sentido de que tendría que contratarse una persona para que se dedique a esta labor, esto trae como consecuencia un gasto económico que habría que estudiar si vale la pena y la otra razón es que varía según la persona que la utilice.

Southwood (1978) y Kretzchmar (1948, citado por Núñez, 1986), indica que la red entomológica es muy sencilla y fácil, pero que tiene la desventaja que hay cambios en la eficiencia en los distintos cultivos. Dumas et al. (1962 y 1964) consideran que la altura de las plantas, factores físicos y el estado del tiempo, influyen en el número de especies predatoras registradas por la red entomológica o por conteos hechos en forma visual. Southwood (1978) estima que la red entomológica no puede medir los cambios en la distribución vertical con respecto a la especie.

\section{CONCLUSIONES}

1- La temperatura es un factor muy importante que influye directamente en la actividad de Liriomyza.

2- Hay diferencia altamente significativa entre todas las trampas y cada una ayuda a predecir comportamientos particulares de la mosca en algún momento dado, según se esté dando el ciclo. La red 1800 captura moscas que se encuentran posadas y sobrevolando el cultivo. Con la red 900 se captura moscas desde la base hasta la parte superior de la planta, puede predecir el comportamiento que tiene la mosca en forma vertical con respecto a la posición en la trampa. Las tarjetas amarillas ayudan a predecir el comportamiento de la mosca en el aspecto vertical del cultivo de papa y determinar en cual estrato pasa más tiempo.

Para efectos de monitorero se recomiendan todas las trampas estudiadas. Para ayudar a bajar la población se pueden usar las trampas de galón amarillas ya que servirían como método cultural, para la captura de moscas. 
3- Los períodos de prefloración hasta cosecha fue donde se encontró la mayor población de L. huidobrensis.

Se encontró que en período de emergencia a, prefloración, la trampa amarilla mostró la mayor captura y se dio a las 7 y 8 am y a las 4 pm.

En el período de prefloración a floración, la de mayor captura fue la trampa amarilla también, a las 9 am, 12 $\mathrm{m}$ y de 3 a 5 pm.

En el período de floración a cosecha, la trampa amarilla muestra su mayor captura desde las 8 am a las $12 \mathrm{~m}$ y 2 a 4 pm.

Con las otras trampas no se muestran picos importantes de máxima captura, tiende haber una fluctuación durante todo el cultivo, estas trampas podrían estar prediciendo el comportamiento real de la mosca minadora a través del ciclo de papa.

\section{LITERATURA CITADA}

ANDREWS, K. L.; QUEZADA, J. R. 1986. Plagas de invertebrados de cultivos en estado actual y potencial en América Central. Editorial el Zamorano. Honduras. 530 p.

CHANDLER, L. D. 1985. Flight activity of Liriomyza trifolii (Diptera: Agromyzidae) in relationship to placement ofyellow traps in bell pepper. Journalof Economic Entomology. 78:825-828.

CHA VES, G. L.; RAMAN, K. V. 1987. Evaluation of trapping and trap types to reduce damage to potatoes by the Leafminer, Liriomyza huidobrensis (Diptera, Agrornyzidae). Insecticidal Science Application. 8(3):369-372.

COMITÉ TÉCNICO DE Liriomyza sp. 1990. El minador de las hojas Liriomyza (Diptera: agromyzidae). Boletín divulgativo No. 95. Ministerio de Agricultura y Ganadería. Centro Agronómico Tropical de Investigación y Enseñanza. Convenio Costarricense Alemán, Sanidad Vegetal-GTZ.26 p.

DUMAS, B. A.; BOYER W. P.; WHITCOMB W.H. 1962. Effect of time of day on surveys of predaceus insects in field crops. Florida Entomology. 45: 121-124
GÓMEZ B. 1992. Tesis de rado: Muestreo y Dinámica Poblacional de Liriomyza huidobrensis (Blanchard) Diptera Agromyzidae en el Cultivo de Papa (Solanum tuberosum). Escuela de Biología, Universidad de Costa Rica. San Pedro de Montes de Oca, San José, Costa Rica. 120 p.

HOLDRIDGE, L. R. 1979. Ecología basadas en zonas de vida. Instituto Interamericano de Ciencias Agrícolas, San José, Costa Rica. 216p.

KRETZCHMAR, G. P. 1948. Soybean insects in Minnesota with special reference to sampling techniques. Journal of Economic Entomology. 41: 586-597.

LOPEZ, C. J. 1986. Disposición espacial, evaluación de daño y dinámica poblacional del picudo del ejote (Apion godmani Wagn), en el cultivo del frijol (Phaseolus vulgaris L.) tesis profesional. México. Universidad Autónoma Chapingo. 43 p.

MATIN, M. A.; YULE, W. N.; MARTEKM P. 1984. Comparison of three methods for sampling northern corn rootworm (Coleoptera: Chrysomellidae) adult populations in two Quebec cornfields. Journal of Economic Entomology. 77:1344-1348.

NUÑEZ BOJÓRQUEZ, R. 1986. Comparación de métodos de muestreo de plagas agrícolas. Seminario 11 (Ent-680) Semestre de Primavera - 1986. Centro de Entomología Acarología, Colegio de Post-graduados. 20 p.

PARRELLA, M. 1984. Effect of temperature on oviposition, freding, and longevity of Liriomyza trifolii (Diptera: Agromyzidae). Canadian Entomologist. 116: 85-92.

PARRELLA, M.; JONES, V. 1984. Coping with the "leafminer crisis". California Agriculture. Sep. 17-20.

PARRELLA, M.; JONES, V. 1985. Yellow traps as monitoring tools for Liriomyza trifolli (Diptera: Agromyzidae) in crysanthemum greenhouses. Journal of Economic Entomology. 78:53-56.

PRICE, J. 1982. An assessment of leafminer manage ment methods on export chrysantemum enterprises in Colombia. South America. Process Florida State Horticultural Society. 95:146-148. 
ROBIN, M.; MITCHELL, W. 1987. Sticky trap for monitoring leafminers Liriomyza sativae and Liriomyza trifolii (Diptera: Agromyzidae) and their associated hymenopterous parasites in watermelon. Journal of Economic Entomology. 80(6):1345-1347.

RODRÍGUEZ, V. C.; ALPIZAR, M. D.; LEÓN, G. R; MONGE, G. J.; CÉSPEDES, Z. R 1990. Evalua-ción de extractos acuosos vegetales en el combate de Liriomyza huidobrensis. Blanchard (Diptero: Agromyzidae) en el cultivo de papa. Hojas mimelografiadas, Departamento de Entomología del Mi-nisterio de Agricultura y Ganadería. Costa Rica. 6p.

RUESINK, W.C.; KOGAN. M. 1975. Quantitative basis of pest management: In Metcalf L. and Luckmann, W. H. eds. Introduction to insect pest Management. New York, Wiley. p 309-51.

SCHUSTER, D. J.; BECK, H. W. 1983. Visual rating system for assessing Liriomyza spp. (Diptera: Agromyzidae) Journal of Economic Entomology. 76: 1465-1466.

SEGURA ORTIZ, E. 1991. Evaluación de insecticidas sobre Liriomyza huidobrensis Blanchard (Diptera: Agromyzidae), su efecto en las polillas y en el rendimiento del cultivo de papa. Tesis Licenciatura. Universidad de Costa Rica, Sede de Occidente. 79 p.
SOUTHWOOD, T. R E.. 1978. Ecological methods, with particular reference to the study of insect populations. 2 ed. Great Britain at the University Printing, House, Cambridge. $375 p$

STEEL, R G. D.; TORRIS, J. H. 1980. Principies and Procedure of statistics. A biometrical approach. 2nd. ed. Mc Graw Hill. New York.

VÉLEZ, A. R; MADRIGAL, A.; MORALES, G. 1980. Biología, hábitos y hospedantes del minador del crisantemo. Revista Colombiana de Entomología. 6:2135 .

ZEHNDER, G. W.; TRUMBLE, J. T. 1985. Sequential sampling plans with fixed levels of precision for Liriomyza species (Diptera: Agromyzidae) in fresh market tomatoes. Journal of Economic Entomology. 78: 138-142.

ZOEBISCH, T. G. 1988. Sampling methods to estimate absolute and relative density of adults and larvae of Liriomyza trifolii (Burgess) on fresh market tomatoes and biology of Liriomyza trifolii under laboratory conditions. Thesis for degree of Doctor of Philosophy. Florida University 163 p. 